\title{
Tie-dyed art in the application of the silk garments
}

\author{
Yugang Chen ${ }^{1, a}$ \\ ${ }^{1}$ Jiangxi Institute of Fashion Technology, Jiangxi, Nanchang, 330201 \\ a28941204@qq.com
}

Keywords: Traditional; Tie-dyed art; Silk garments

\begin{abstract}
Extremely rich traditional tie-dye process with its unique glamour of art has a long history, manual tie-dye tie way of diversity, design of of primitive simplicity is elegant in style, is another popular element. Regarded in the fashion clothing at home and abroad, and the integration of silk, more provides designers with unique artistic style.

\section{The introduction}

Tie-dye is derived from a kind of traditional folk handicraft art, recorded in the ancient books to dye "ground" or "the pattern-dye". And tie-dye crafts is also a kind of traditional dyeing techniques, our tie-dye crafts from today already has more than two thousand years of history, is truly a treasure of the Chinese nation.
\end{abstract}

\section{The concept and characteristics of dye}

Traditional tie-dye crafts can be characterized by two types of tie and dyeing. Through the different tools such as cotton fabrics, cotton, hemp, etc., the will be yarn-dyed fabric, sewing, clamp, different manual techniques, such as comprehensive after dyeing. Effect is made the bundle cannot be dyed fabric, the fabric were part of it with grey fabric, not tied appears the dyeing effect of fabrics part of living. Through after tie-dye color shading on fabric immediately, rich variety of color gradients, and fold the effect of printing and dyeing. Tie-dye usually gives priority to tone with blue and white two colors, blue and white two color comparison can show the character of Chinese traditional simple implicative, on colour of blue and white combination is simple quietly elegant coincides with "traditional blue and white porcelain". Tie-dye process of the selected material is given priority to with more soft and light fabric. According to design good design flat sewing with lines, the main seam techniques have folding seaming, full needle sewing method, etc. While another binding through will be part of the dyed yarn or other materials around, make some failed to dyeing, strapping method still can use splint, packet content method, line binding method and so on.

Tie-dye applied in modern textile design, costume design, fiber art design, was a lot of common used in hanging, silk scarves and costumes. Tie-dye process reflects the typical characteristics of folk art, traditional designs is the epitome of the Chinese nation, enrichment of traditional regional customs and different style, but also with other technology to constitute the unique charm of dyeing culture, modern design combined with tie-dye, reflects the new concept of art and design.

Because the traditional dyeing method and technique, a long time to dye the color is plain blue and white color is given priority to, simple line electricity, modelling are the basic building blocks of its design elements. To present a simple elegant artistic style. $60 \mathrm{~s}$ to $90 \mathrm{~s}$ last century, western new design ideas, let dyeing crafts great changes have taken place. On the color changed the single, showed a different colour is given priority to, and on the design modelling also extremely has the western modern abstract style. And tie-dye art now has developed into the integration of different kinds of style, expression of realism, rustic style, fashion style, etc. And in recent years at home and abroad many large fashion show, tie-dye art often be applied by the designer in the fashion design. Such as han silks cup in 2009, the 2009 Beijing fashion week fur, D\&G, 2010 women's wear in the spring and summer clothing design works are find tie-dyed art design. Through successful clothing design work, can reflect the tie-dyed art unique craft technique now has formed new fashion clothing, clothing style unique show. 


\section{The process features of dye on silk fabric}

Removal of soft cotton and linen fabrics, quality noble silk fabrics are tie-dye process in the choice of important materials, silk fabrics usually includes silk, tussah silk, castor silk, cassava, silk, etc. Silk fabrics mainly divides into the kinds of crepe DE chine, heavy crepe georgette burnt-out, georgette, double Joe, Joe, \{sang pu\} satin, crepe, satin elastic crepe satin, several kinds of warp knitting, etc. And most of the natural silk is the white, also have special varieties, mulberry silk is the most precious species in wild silkworm silk, show jade. However, these color for dress design or insufficient performance greatly. To the design of the color mainly embodied in dyeing and printing two kinds big.

The innovation of the design elements designed for fabric again, the new fabric design formed in silk fabric dyeing design performance of two kinds of extremely prominent fashion design language, and the effect of dyeing silk design better conform to the general trend of the culture of popular features. Using various clothing materials, different materials are perfect combination collocation gives a rich and unique artistic effect. At the same time, using silk dyed fabric appearance, texture difference, diversity, combined with a variety of design methods and style at the same time, using the principle of color harmony or contrast color contrast, to show the full colorful or harmonious and unified. Typically we miss manners garment design in the 08 Beijing Olympic Games can reflect to dye the silk "dignified elegance", the designers use quite a lot of Chinese elements in the design, fabric selection is silk, each design reflects the antique flavor, exquisite traditional embroidery technics, knot button, color also is stylist clever application, the performance of the Oriental female elegant protruding to pour from the cause.

\section{Tie-dye process characteristic performance design application in silk garments}

Today's clothing market booming, tie-dye pattern has not stopped on the traditional pattern, pattern is more and more diverse. In our today's life such as tie-dye in a high-grade process performance, are widely used in the design of fashion, after the designer of clever conception, use float soft texture of natural silk fabrics, color and pattern of clothing creation, make people feel stretch, fluent, with unique national characteristics. Tie-dye performance is now not only appear in the traditional pure cotton cloth, tie-dye pattern has appeared in the all kinds of natural and synthetic materials, silk, linen, leather, knitting, denim made of tie-dye effects of all kinds.

The performance of clothing on the local. On local we can show the design of the main means in some parts of the clothing seat through the tie-dye process technique performance design effect, thereby can, to a lot of different pattern texture shape and a variety of colours. It is often placed at the edge of the garment and the center position. Like in the evening the shoulder and waist decoration tie-dyed patterns, will have a dazzling visual effects. Different parts, when using tie-dye process should be different, edge, should choose some graphics simple process, simple color, of course, a single is better, to get strong effect of eye-catching appearance. Near the center of color change, scale is larger, the decorative pattern is often in the form below. Choose more design concrete or abstract. Process can choose to sew, impregnation process, such as color can choose a variety of free collocation.

Garments' integral design of the application. Traditional tie-dye crafts in most of the integral design of the clothing is a alone or in combination with a variety of craft method to create a design. Such as simple a strapping of dyeing technique applied in the overall clothing design, the effect is very good highlights the visual effect of clothing. By combining different methods of several kinds of arts and crafts design method, is more artistic expression, can present rich color, more visible in the haute couture.

Background of clothing design. Background of clothing design is the application of the method of dyeing crafts to the local and the overall design of the clothing. The designed pattern directly show the clothing in the background, also can be combined with some spelling process method, combination is unique characteristics, stylized visual appearance. Tie-dye process as the background of clothing decorated with difficulty usually choose when is low, single technology is 
advisable.

Haute couture to dye the garment dye to mainly is divided into graphic design, material selection, process technique application so several parts. To traditional folk tie-dye this folk art is clever and a perfect fusion of contemporary and contracted fashion clothing both together produced by chemical reaction, two levels of differentiation, will let you get the feeling that find everything new and fresh. Say we are going to a fashion style using the typical traditional tie-dye patterns, while retaining the characteristics of the clothing style and the color is popular now, we can imagine this dress contrast application can make this dress more fashionable.

If the tie-dye technology combined with modern one of the most popular clothing fabrics, or treated with tie-dye techniques to deal with the design of fabrics and other fabrics can be mixed together, can also be promoted in the modern clothing tie-dye garment fashion and popularity. We can also through two models of ready-to-wear clothing, among them the key hair dye in a costume design and process performance, this can coruscate gives outstanding garment immediately. We can also use will be a haven't cut the fabric through tie-dye process decoration in the first place, then according to the effect of dyeing and finishing garment making, so also can achieve the result of having a unique style. Make various process to match the style of tie-dye, tend to make the design more style. Modern garment using so many different crafts tie-dye techniques, is hoping to rich dress design style changes, lets tie-dyed art in clothing from design through the collocation of color have higher development and new changes.

\section{Conclusion}

Our traditional tie-dye is the precious resources of the folk art, is worth to spread the classic, in the long history of several thousand years, tie-dye art has a glorious past. Also once in the time of the annual rings of he had been forgotten and neglected, but our folk art with strong vitality, in today's design, show a different style. We have been know tie-dye process is relatively simple but not simple, while watching it on process performance is relatively simple but still want to get a good effect for aesthetic needs diversification and individuation, to form a perfect work. So, we in the contemporary fashion design of dyeing process on the performance of traditional often special, unique, with this peculiar charm effects affecting the modern people's life, at the same time satisfy the modern people's pursuit of personal taste and yearning.

With the development of era, the traditional culture has been praised by the people's attention and praise highly, people also more and more eager to know their national traditional culture and art, so the tie-dye and combining technology is more and more people like to our life, the traditional tie-dye technology and high-grade silk fabric is characteristic of together, to the innovation of a new a personalized handicraft fabric, we want to inherit preciously and treasure to our application has been passed down, let the child children ancestors can enjoy the essence of traditional craft. We should fully will carry forward the advantages of our national, make the garment more artistic, popularity and fashion.

\section{Reference}

[1] Li Hui,Mingzhen Chen. The artistic features of modern tie-dye [J]. Journal of textile science and technology progress. 2009, 8.

[2] Lili xia,Xiaohang Zhu. Analyze the tie-dyed art beauty [J]. Journal of art. 2012, 6.

[3] Minhua Yang,SiWei. Modern tie-dye art performance in the clothing design [J]. Journal of foreign silk. $2007,6$. 